\title{
A Novel Model of Obesity-Related Diabetes: Introgression of the Lepria Allele of the Zucker Fatty Rat into Nonobese Spontaneously Diabetic Torii (SDT) Rats
}

\author{
Taku MASUYAMA ${ }^{1,2)}$, Yoshiaki KATSUDA ${ }^{2,3)}$, and Masami SHINOHARA ${ }^{2)}$ \\ 1)Toxicology Research Laboratories, Central Pharmaceutical Institute, JAPAN TOBACCO Inc., 23 \\ Naganuki, Hadano-shi, Kanagawa 257-0024, ${ }^{2}$ Torii Pharmaceutical Co. Ltd., 3-4-1 Nihonbashi- \\ honcho, Chuo-ku, Tokyo 103-8439, and ${ }^{3}$ Biology Research Laboratories, Central Pharmaceutical \\ Institute, JAPAN TOBACCO Inc., 1-1 Murasaki-cho, Takatsuki-shi, Osaka 569-1125, Japan
}

\begin{abstract}
An fa allele of the leptin receptor gene (Leprfa) of the Zucker fatty rat was introduced into the genome of the Spontaneously Diabetic Torii (SDT) rat, an inbred model of nonobese type 2 diabetes mellitus, through the 'Speed congenic method'. The newly established congenic strain of a SDT rat for Leprfa was maintained by intercrossing between fa-heterozygous littermates, and the phenotypes related to obesity and diabetes were investigated till 32 wks of age. SDT fa/fa rats of both sexes exhibited obesity, adiposity and insulin resistance associated with hyperphagia from the loss of leptin action. Interestingly, they developed diabetes from 5 wks of age in males and 8 wks in females with the incidences reaching $100 \%$ at 16 wks in males and $73 \%$ at 32 wks in females. In contrast, heterozygous (+/fa) and wild-type (+/+) rats developed spontaneous nonobese diabetes in males from approximately $20 \mathrm{wks}$, but not in females, as with the original SDT rats. These results indicate that the fa gene accelerates the onset of diabetes in SDT rats by making adiposity and/or insulin resistance as potent risk factors for development of their diabetes. The SDT.Leprfa congenic rat strain is expected to be a novel model of obesity-related diabetes and could be a useful tool for studies of the genetic backgrounds of diabetes in response to fa-induced obesity.
\end{abstract}

Key words: congenic strain, diabetes, fa, obesity, SDT rat

\section{Introduction}

The Spontaneously Diabetic Torii (SDT) rat is known as a useful model of nonobese type 2 diabetes that spontaneously develops hyperglycemia and glucose intolerance in males resulting from decreased insulin secretion due to $\beta$-cell degeneration leading to inadequate $\beta$-cell mass in the pancreas [12,17]. In addition, the diabetes in SDT rats is a polygenetic trait controlled by at least 3 quantitative trait loci (QTLs), Gisdtl, Gisdt2 and Gisdt3, localized on chromosomes 1, 2 and $\mathrm{X}$, respectively [11]. Thus, it is clear that the SDT rat

(Received 29 June 2004 / Accepted 21 September 2004)

*Address corresponding: T. Masuyama, Toxicology Research Laboratories, Central Pharmaceutical Institute, JAPAN TOBACCO Inc., 23 Naganuki, Hadano-shi, Kanagawa 257-0024, Japan 
has a genetic background which produces glucose intolerance associated with decreased insulin secretory capacity.

Type 2 diabetes mellitus is a polygenic disorder that is caused by a metabolic and/or hormonal imbalance between insulin secretion from $\beta$-cells and insulin sensitivity in peripheral tissues both of which might be modified by genetic and environmental factors $[14,20]$. The decreased sensitivity to insulin leads to an increased requirement for insulin, and is often associated with obesity in which metabolic disturbances are marked in insulin-target organs, such as the liver, muscle and adipose tissues $[1,14]$. It is well known that the $\beta$-cell response to increased insulin requirements is strongly determined by animals' genetic backgrounds [3, 19, 21].

Based on the above concept, it should be possible to establish a novel model of obesity-related diabetes by introducing the genetic factor of insulin resistance or obesity into the genetic background of the SDT rat. The genetic factor that produces insulin resistance or obesity, an $f a$ mutation of the leptin receptor gene $\left(\operatorname{Lepr}^{f a}\right)$ in the Zucker fatty (ZF) rat, is well known [2]. The leptin receptor is mainly expressed in the hypothalamus and is a key molecule that regulates hypothalamic effects on satiety and energy expenditure through binding to leptin, an adipocyte-specific hormone $[16,22]$. The fatty phenotype in the ZF $(f a / f a)$ rat is characterized by obesity resulting from a defect in appetite control [2] which is caused by an $f a$ mutation in the Lepr gene, a missense mutation (an A-to-C conversion at nucleotide position 806) in a MspI site that results in a single amino acid change from $g \ln$ to pro at position $269[2,18]$.

We thus established a congenic line of the SDT rat by introducing the $f a$ allele of the ZF rat into the SDT rat genome through the 'Speed Congenic Method' popularized by Markel et al. [9] using a PCR technique with DNA markers. In this paper, we describe the process of the establishment of a new congenic strain of the SDT rat and the clinical features related to obesity and diabetes of this congenic strain.

\section{Materials and Methods}

\section{Animals and animal handling}

SDT rats, the recipient strain, were used from our colony. The ZF rat, the donor of the $f a$ allele of the
Lepr gene, was provided by Prof. Komeda of Tokyo Medical University (Tokyo, Japan). Animals were individually housed in a room maintained at $23 \pm 1{ }^{\circ} \mathrm{C}$ and $55 \pm 10 \%$ relative humidity with a 12-h light-dark cycle, and were provided tap water (sterilized by UV light) and food (CRF-1, Charles River Japan Inc., Kanagawa, Japan) ad libitum, except when they were fasted for experiments. All the investigations were approved by committees for the humane care and use of animals in our laboratories, in accordance with the Standards Relating to the Care and Management of Experimental Animals (Notification No.6, March 27, 1980 of the Prime Minister's Office in Japan).

\section{Introgression of the fa gene to the SDT rat}

Introgression of $f a$ gene onto the SDT rat genome was carried out according to a 'Speed Congenic Method' [9], which is a backcrossing of the fa-carriers to the recipient SDT rats. Briefly, the $f a$-carriers that were produced by first mating between female SDT rats and one male heterozygous $(+/ f a)$ ZF rat were subsequently backcrossed. The rats for the next generation production were selected from the $f a$ allele-carrying progenies with the lowest heterozygous segments based on the genotypes of simple sequence-length polymorphism (SSLP) markers across the entire genome. Congenic rats introgressed with the $f a$-alleles were maintained by intercrossing between heterozygous littermates $(+/ f a)$ to obtain rats having each genotype, $+/+$, $+/ f a$ and $f a l f a$.

\section{Genetic monitoring}

SSLP markers were purchased from Research Genetics Inc. (Huntsville, Ala, US) or synthesized based on the sequences published on websites (The Rat Genome Database RatMap, http://ratmap.gen.gu.se/), to analyze the polymorphism of the PCR products between the ZF rat and the SDT rat. A total of 226 SSLP markers were selected covering all of the chromosomes, with an average span of $6.2 \mathrm{cM}$ (corresponding to approximately $1,500 \mathrm{cM}$ in total), in order to monitor heterozygosity in the background genome of the backcrossed rats. Genomic DNA extraction, PCR and electrophoresis were performed as described previously [10].

Genotyping of the $f a$ locus was performed according to a PCR-restricted fragment length polymorphism (RFLP) method as follows. A primer set to detect the $f a$ 
allele was designed to cover a 110-bp region containing the $f a$ mutation site in the Lepr gene with the following sequences: 5'-TATGGAAGTCACAGATGATGG-3' and 5'-CTTACGATTGTAGAATTCTCTAA -3'. This PCR product (110 bp) was digested by a restriction endonuclease $\mathrm{MspI}$ (Nippon Gene Co., Toyama, Japan) at $37^{\circ} \mathrm{C}$ for $1 \mathrm{~h}$ to cut the $f a$ allele, and then electrophoresed on a 4\% agarose gel (NuSieve 3:1 agarose, FMC BioProducts Inc., MA, U.S.A.) for genotyping. The MspI digestion of the $f a$ allele produces two fragments of 77 and $33 \mathrm{bps}$.

\section{Phenotypic characterizations}

The animals bred for this study were routinely checked for the appearance of glucosuria using Multi-Sticks (Bayer-Sankyo, Tokyo, Japan) from 4 to 32 weeks of age. The diagnostic criteria for diabetes in this study were the appearance of glucosuria and hyperglycemia (fed blood glucose $\geq 250 \mathrm{mg} / \mathrm{dl}$ ). At 4, 5, 6, 8, 10, 12, $14,16,20,25$ and 32 weeks of age, the animals were weighed, and their blood glucose concentrations were determined under non-fasted conditions using a portable glucose meter (DexterZ, Bayer-Sankyo, Tokyo, Japan) within $1-4 \mathrm{~h}$ after the light cycle began. Food consumption was measured at 6 weeks of age. At 14 weeks of age, after overnight fasting, animals were ether-anesthetized, weighed, measured for their body length (from anus to nose), and exsanguinated from the abdominal aorta. Retroperitoneal and interscapular fat pads were removed and weighed. Blood samples collected into heparinized tubes were centrifuged to obtain the supernatant plasma. The plasma glucose concentrations were determined by the glucose oxidase method using an autoanalyzer (HITACHI 7150, HITACHI, Tokyo, Japan) with reagents from Wako Chemical Inc. (Osaka, Japan). The concentrations of plasma insulin and leptin as an immuno-reactant were determined by an enzyme-linked immunosorbent assay (ELISA) using commercially available kits (Morinaga Biochemical Research Laboratory, Yokohama, Japan) for rat insulin and leptin. Body mass index (BMI, $\mathrm{g} / \mathrm{cm}^{2}$ ) was determined by dividing body weight $(\mathrm{g})$ by the square of body length $\left(\mathrm{cm}^{2}\right)$.

\section{Statistical analysis}

The data are shown as means \pm standard deviations (S.D.). The statistical differences between $f a / f a$ and $+/$ + rats were determined by Student's $t$-test.

\section{Results}

\section{Establishment of the congenic strain}

First, female SDT rats were crossed with a male heterozygous $(+/ f a) \mathrm{ZF}$ rat to obtain the F1 hybrids carrying the $f a$ allele. The heterozygous F1 females $(+/ f a)$ were backcrossed to male SDT rats, and the N2 generation was obtained. Subsequently, backcross mating was done between the progenies and the recipient SDT rats. In the N5 generation, we obtained 'the best male' in which all of the SSLP markers covering the entire rat genome were genotyped to SDT-homozygous, except for D5Rat159 flanking Lepr. The genetic distance between D5Rat159 and Lepr was $4 \mathrm{cM}$ on the Rat Genome Database RatMap (Table 1). We conducted further backcrosses until the N7 generation. Although 130 male fa-carriers were produced among N2-N7 generations, we could not find any with recombination between D5Rat159 and Lepr, suggesting that both loci are closely adjacent at a distance of less than $0.8 \mathrm{cM}$ in this cross. The maximum genetic length of a heterozygous interval was estimated to be $6 \mathrm{cM}$ in $63-69 \mathrm{cM}$ of chromosome 5 (Table 1). The heterozygous interval was considered to be less than approximately $0.3 \%$ of the entire rat genome $(2,028 \mathrm{cM}$ on the KLAS map (Kyoto Laboratory Animal Science map)) [8]. At present, this congenic strain is maintained by inter-crossing between $f a$-heterozygous littermates.

\section{Obesity-related phenotypes in congenic rats}

Gross appearances of the 6 -wk-old male SDT $+/+,+/$ $f a$ and $f a / f a$ rats are shown in Fig. 1. The $f a / f a$ rats became overtly obese and were distinguishable from wild-type (+/+) and heterozygous (+/fa) lean rats.

Their growth curves until 32 weeks of age are shown in Figs. 2A and 2B. The body weights of the SDT-fal $f a$ rats of both sexes were higher than those of the wildtype $(+/+)$ and heterozygous $(+/ f a)$ lean rats. The mean peak body weight of the falfa rats reached the maximum value: $694.9 \mathrm{~g}$ for males (at $20 \mathrm{wk}$ ) and $708 \mathrm{~g}$ for females (at $32 \mathrm{wk}$ in final observation), 1.5-fold and 1.7-fold higher than age-matched wild-type rats, respectively. Wild type (+/+) and heterozygous $(+/ f a)$ lean rats showed similar growth curves to the original SDT rats as reported previously [17]. Also, the BMI as an index of obesity was statistically greater $(P<0.01)$ in $f a /$ $f a$ rats of both sexes $\left(0.91\right.$ and $0.87 \mathrm{~g} / \mathrm{cm}^{2}$ in males and 
Table 1. Genetic monitoring of rat chromosome 5 in SDT.Lepr ${ }^{f a}$ congenic rats

\begin{tabular}{|c|c|c|c|c|}
\hline $\mathrm{cM}^{\mathrm{a})}$ & Locus & & & \\
\hline 0 & D5Rat116 & & & \\
\hline 7 & $\mathrm{D} 5 \mathrm{Mgh} 2$ & & & \\
\hline 12 & D5Rat123 & & & \\
\hline 18 & D5Mgh1 & & & \\
\hline 19 & D5Rat126 & & & \\
\hline 41 & D5Rat8 & D5Mit1 & & \\
\hline 44 & D5Rat12 & D5Mit2 & & \\
\hline 45 & D5Rat104 & D5Mgh6 & & \\
\hline 51 & D5Rat85 & D5Rat20 & D5Mit11 & \\
\hline 52 & D5Rat19 & & & \\
\hline 54 & D5Rat 150 & D5Rat149 & & \\
\hline 56 & D5Wox3 & D5Rat98 & D5Rat92 & D5Rat88 \\
\hline 58 & D5Rat24 & & & \\
\hline 62 & D5Rat154 & & & \\
\hline 63 & D5Rat71 & D5Rat156 & D5Rat155 & \\
\hline 65 & Lepr* & & & \\
\hline 69 & D5Rat159* & D5Rat27 & & \\
\hline 71 & D5Rat95 & D5Rat65 & & \\
\hline 72 & D5Rat165 & D5Rat108 & & \\
\hline 78 & D5Rat34 & D5Rat33 & D5Rat203 & \\
\hline 80 & D5Rat36 & & & \\
\hline 86 & D5Rat89 & D5Rat43 & D5Rat37 & \\
\hline 88 & D5Rat39 & D5Mgh8 & D5Mgh14 & \\
\hline 92 & D5Rat93 & D5Rat69 & & \\
\hline 93 & D5Rat41 & & & \\
\hline 97 & D5Rat56 & & & \\
\hline 103 & D5Rat47 & & & \\
\hline 105 & D5Mit15 & & & \\
\hline 106 & D5Rat48 & & & \\
\hline 109 & D5Mgh16 & D5Mgh15 & & \\
\hline
\end{tabular}

a) Genetic distance of each locus was based on the integrated linkage map of the Rat Genome Database RatMap. Asterisks indicate loci heterozygous in the N7 backcrossed generation. Maximum genetic length of a heterozygous interval was estimated to be $6 \mathrm{cM}$ in the $63-69 \mathrm{cM}$ of chromosome 5 .

females, respectively) than in lean rats $(0.75$ and 0.58 $\mathrm{g} / \mathrm{cm}^{2}$ in males and females, respectively) at 14 weeks of age (Figs. 2C and 2D). Retroperitoneal and interscapular fat pads, as indexes of adiposity, were markedly heavier in falfa rats of both sexes $(26.2$ and $10.1 \mathrm{~g}$, respectively, in males and 29.1 and $16.6 \mathrm{~g}$, respectively, in females) than in wild-type $(+/+)$ rats $(11.0$ and $1.9 \mathrm{~g}$, respectively, in males and 8.2 and $1.5 \mathrm{~g}$, respectively, in females) (Figs. 2E and 2F). Food consumption in fa/fa rats of both sexes was significantly increased $(P<0.01)$ to approximately double at 8 weeks compared with that in lean rats (Figs. $2 \mathrm{G}$ and $2 \mathrm{H}$ ).

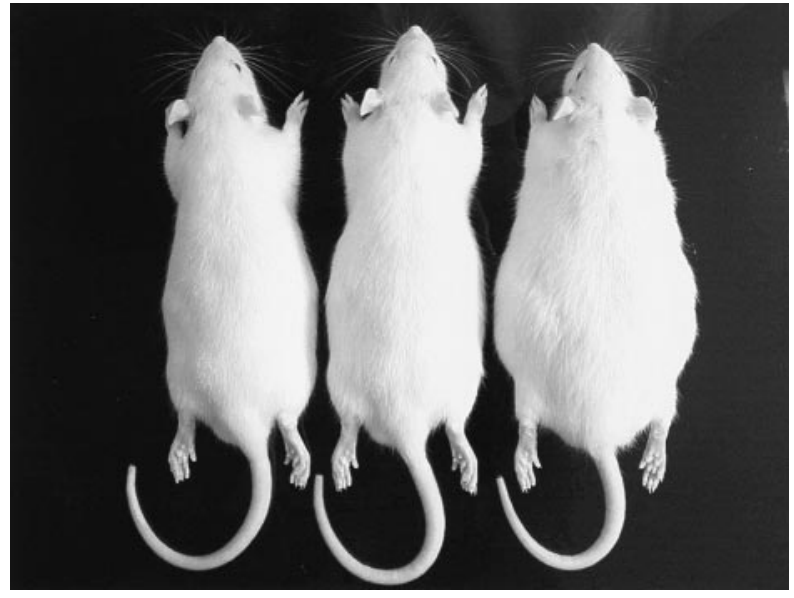

Fig. 1. The dorsal appearance of male SDT.Leprfa congenic rats at 6 weeks of age. Littermates of +/+ (left), +/fa (middle) and falfa (right) are shown.

Male and female heterozygous (+/fa) and wild-type $(+/+)$ rats showed no differences in the above phenotypes during their life span.

\section{Diabetes-related phenotypes in congenic rats}

The cumulative incidence of the onset of diabetes (glucosuria and hyperglycemia) is shown in Figs. 3A and $3 \mathrm{~B}$. Male falfa rats developed diabetes from 5 weeks of age, with the cumulative incidence of diabetes reaching up to $82 \%$ at 8 weeks of age and $100 \%$ by 16 weeks of age (Fig. 3A). Female falfa rats developed diabetes with a lower incidence $(73 \%$ at 32 weeks of age) than the male $f a / f a$ rats (Fig. 3B). In contrast to falfa rats, +/+ and +/fa rats of both sexes showed similar phenotypes to the original SDT rats as reported previously $[11,12,17]$. Male $+/+$ and $+/ f a$ rats exhibited delayed onset of diabetes (18 weeks) and the incidence reached $100 \%$ at 29 weeks of age. Female $+/$ + and $+/ f a$ rats did not develop diabetes until 32 weeks of age (Figs. 3A and 3B).

Changes in blood glucose levels under non-fasting conditions are shown in Figs. 3C and 4D. The male $f a /$ $\mathrm{fa}$ rats showed higher blood glucose levels than the male lean $(+/+,+/ f a)$ rats after 6 weeks of age, and became more hyperglycemic by 8 weeks of age (Fig. $3 \mathrm{C})$. The lean male rats were hyperglycemic after 20 weeks of age (Fig. 3C), as with the original SDT rats [17]. In female $f a / f a$ rats, the average blood glucose levels were slightly but significantly elevated after 6 

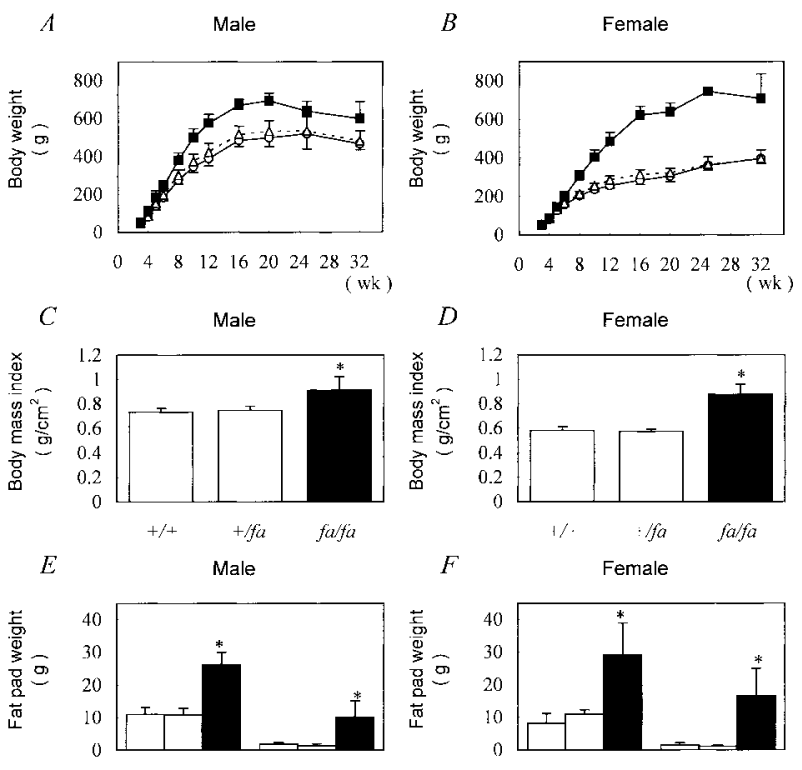$$
F
$$

Female
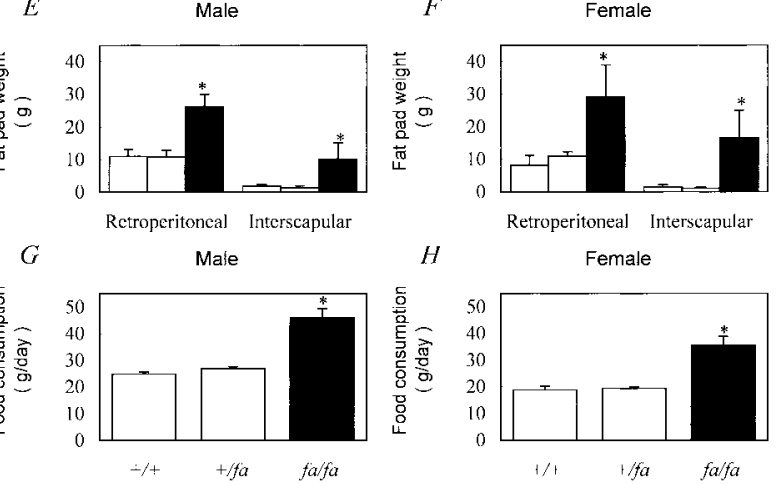
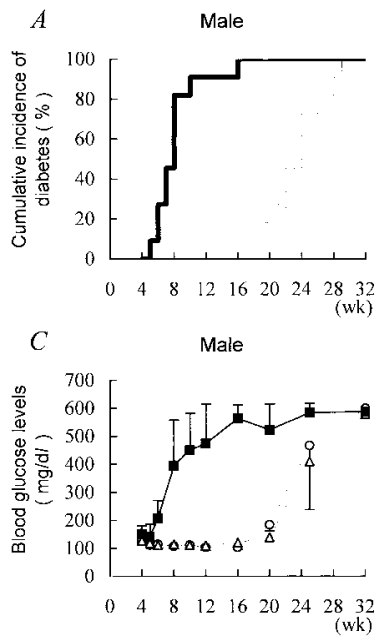
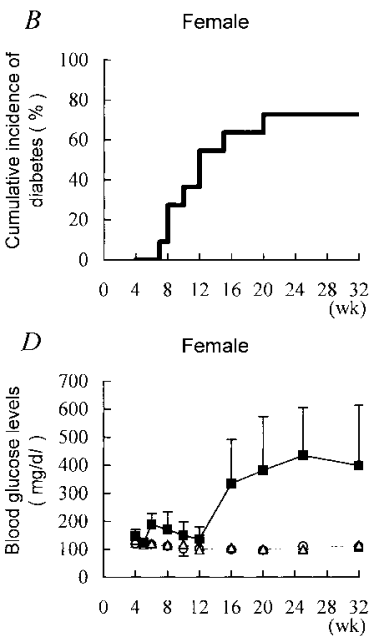

Fig. 3. Diabetes-related phenotypes in SDT.Lepr ${ }^{f a}$ congenic rats. Cumulative incidence of diabetes development in males (A) and females (B) until 32 weeks of age; bold lines, $f a / f a$ homozygous rats (males $=11$, females $=11$ ); dotted lines, + / $f a$ heterozygous rats (males $=20$, females $=4$ ); solid lines, + / + wild-type rats (males $=11$, females $=17)$. The cumulative incidence in female $+/+$ and $+/ f a$ rats was $0 \%$ till 32 weeks of age, as hidden by $\mathrm{X}$-axis indicating age in weeks. Changes in blood glucose levels until 32 weeks of age in males (C) and females (D); black boxes, falfa homozygous rats; white triangles with dotted lines, $+/ f a$ heterozygous rats; white circles, +/+ wild-type rats. Data are expressed as the mean \pm S.D. for each point Statistical differences $(P<0.01)$ between $f a / f a$ and $+/+$ rats were observed from 6 to 20 weeks of age in males and after 6 weeks of age in females.

\section{Discussion}

In this study, we established a new congenic rat strain produced by introgression of the $f a$ allele of a ZF rat onto the SDT rat genome. This congenic rat spontaneously develops diabetes associated with obesity in $f a$-homozygous rats.

The $f a$ mutation in the rat leptin receptor accounts for the fatty phenotype in ZF falfa rats resulting from impaired appetite control [2]. In this study, SDT falfa rats of both sexes exhibited obesity with adiposity. Hyperphagia and hyperleptinemia were also observed in the falfa rats of both sexes as compared with the wild-type and heterozygous lean rats, suggesting that impaired appetite control by leptin resistance in the hypothalamus exists in these rats. This indicates that the $f a$ gene evoked a similar phenotype in the SDT background as observed in $\mathrm{ZF}$ rats. 

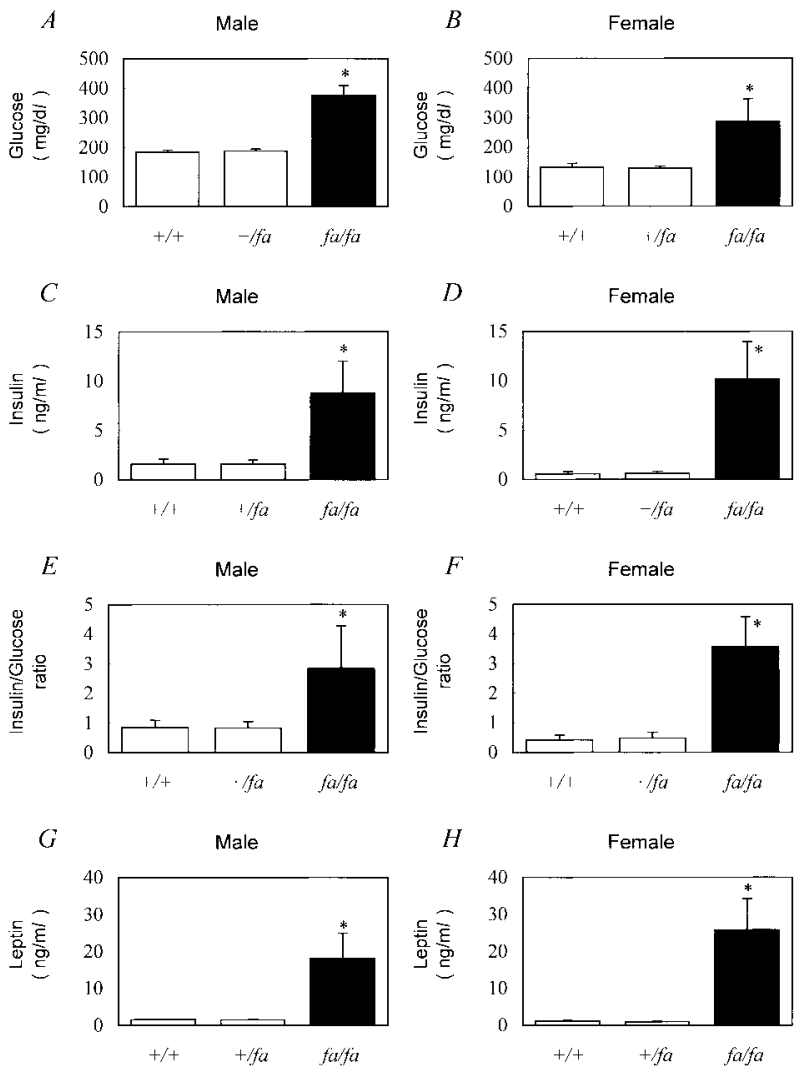

Fig. 4. Plasma biochemistry of fasted SDT.Leprfa congenic rats at 14 weeks of age. Glucose (A, B), insulin (C, D) and leptin $(\mathrm{G}, \mathrm{H})$ concentrations in plasma and insulin/glucose ratio $(\mathrm{ng} / \mathrm{mg})$ in $1 \mathrm{ml}$ plasma $(\mathrm{E}, \mathrm{F})$ of males $(\mathrm{A}, \mathrm{C}, \mathrm{E}$ and $\mathrm{G}$ ) and females (B, D, F and H) are shown. Data are expressed as the mean \pm S.D. of 4-6 rats for each genotype. Asterisks indicate statistical differences $(P<0.01)$ to the wild-type.

In contrast, ZF fa/fa rats of both sexes do not develop diabetes during their lifespan when fed a normal chow ad libitum in similar environmental conditions to those used in our investigations [3, 15, 23], although they show an impaired glucose tolerance and other signs of insulin resistance associated with obesity. We found that SDT $\mathrm{fa} / \mathrm{fa}$ rats spontaneously developed hyperglycemia after 5 weeks of age in males and after 8 weeks of age in females, the onset of which is markedly earlier than in wild-type and heterozygous rats. The cumulative incidence reached $100 \%$ in males by 16 weeks of age and $73 \%$ in females at 32 weeks of age. These results indicate that the $f a$ gene accelerates the onset of diabetes in SDT rats. SDT $f a / f a$ rats had higher fasting plasma insulin concentrations with an increased insulin/glucose ratio than did lean rats, suggesting that they had increased insulin resistance. Insulin resistance is a major risk factor and diabetes develops only when $\beta$-cells fail to compensate for increased insulin demand due to hormone resistance [21]. Inadequate $\beta$ cell mass is found in SDT rats [12], which appears to be determined by the genetic background of these congenic rats. Thus, the obesity and/or insulin resistance induced by the $f a$ gene are regarded as potent risk factors for accelerating diabetes development in SDT rats.

Both the wild-type (+/+) and heterozygous (+/fa) rats showed no significant differences in the characteristics of obesity- and diabetes-related phenotypes, and exhibited spontaneous diabetes without obesity after 18 weeks of age in males, while the females were nondiabetic at least until 32 weeks of age (the end of the observation period). Their age-related phenotypes were similar to those in the recipient SDT rats. This is the biological evidence that the genetic background of this congenic strain was sufficiently replaced by the SDT rat genome. In addition, no effect of the heterozygosity for the $f a$ allele is expected to be in the SDT rat genome as well as in the Zucker background.

Although a large number of rat strains having the Lepr gene mutation have been reported [4-6, 15], the SDT- $f a$ congenic strain we established is important in terms of having the genetic background of the nonobese type 2 diabetes of the SDT rat. Diabetic phenotypes of SDT $f a / f a$ rats described in this study may partially resemble those of Zucker Diabetic Fatty ( $f a / f a$ ) rats exhibiting a severe form of obese diabetes [15]. For example, both $\mathrm{fa} / \mathrm{fa}$ males of SDT and ZDF rats display similar magnitudes of glycemia and insulinemia; blood glucose levels and plasma insulin concentrations were $474.8 \pm 140.4 \mathrm{mg} / \mathrm{dl}$ and $8.79 \pm 3.22 \mathrm{ng} / \mathrm{ml}$, respectively, in the SDT $\mathrm{fa} / \mathrm{fa}$ rats at $12-14$ weeks of age (Figs. 3C and 4C) and $504 \pm 84 \mathrm{mg} / \mathrm{dl}$ and $8.8 \pm 3.0$ $\mathrm{ng} / \mathrm{ml}$ in ZDF fa/fa rats at 10-13 weeks of age as reported by Peterson et al. [15]. However, the SDT background contains genetic defects which impair insulin secretory capacity to glucose stimuli rather than insulin resistance [11]. Further characterization of this congenic rat might be required not only to define a phenotypic difference among the several obese diabetes models but also to understand the heterogeneous pathology of obesity-related diabetes involved with genetic combinations of the SDT-background-derived 
defects and the Lepr gene mutation.

It is well known that genetic background strongly influences susceptibility to diabetes [3, 13, 19]. Chung et al. studied two rat models of diabetic and non-diabetic strains having the Lepr ${ }^{f a}$ mutation [3] and determined three QTLs for the variability in the susceptibility to obese diabetes [3]. Of these QTLs, interestingly, the locus regulating adiposity and the number of pancreatic islets was mapped on chromosome 1, positionally overlapping a highly significant QTL, Gisdt1, for glucose intolerance in the SDT rat [11]. The SDT- $f a$ congenic rat might contribute to the identification of the susceptibility genes to diabetes with or without obesity.

In summary, we established a novel congenic rat strain of the SDT rat by introgression of Lepr ${ }^{f a}$ into the SDT rat genome resulting in obesity due to polyphagia. This congenic rat strain might be a useful tool for studying the underlying mechanisms of diabetes development with or without obesity. At present, this congenic strain is maintained as a segregating inbred strain for Lepr ${ }^{f a}$ by intercrossing (sister-brother mating). This congenic strain has been designated 'SDT.Cg-Lepr ${ }^{f a}$ ' according to the rules for the Nomenclature of Mouse and Rat Strains, because the ZF rat used as the donor for Lepr fa was an 'outbred' strain. In practice we call it the 'SDT fatty rat', in the same manner as other fatty rats having the Lepr ${ }^{f a}$ mutation such as the Zucker fatty, Zucker diabetic fatty (ZDF) and Wistar fatty.

\section{Acknowledgments}

The authors thank to Prof. Kajuro Komeda (Tokyo Medical University) who passed away in Jan. 2003, because he kindly requested us to carry out this work and also provided a male Zucker fatty $(+/ f a)$ rat. We also thank all of the staff of our laboratories for technical support and animal care.

\section{References}

1. Cavaghan, M.K., Ehrmann, D.A., and Polonsky, K.S. 2000. Interactions between insulin resistance and insulin secretion in the development of glucose intolerance. J. Clin. Invest. 106: 329-333.

2. Chua, S.C. Jr., White, D.W., Wu-Peng, X.S., Liu, S.M., Okada, N., Kershaw, E.E., Chung, W.K., Power-Kehoe, L., Chua, M., Tartaglia, L.A., and Leibel, R.L. 1996. Phenotype of fatty due to Gln269Pro mutation in the leptin receptor (Lepr). Diabetes 45: 1141-1143.

3. Chung, W.K., Zheng, M., Chua, M., Kershaw, E., PowerKehoe, L., Tsuji, M., Wu-Peng, X.S., Williams, J., Chua, S.C. Jr., and Leibel, R.L. 1997. Genetic modifiers of Lepr fa associated with variability in insulin production and susceptibility to NIDDM. Genomics 41: 332-344.

4. Guberski, D.L., Butler, L., Manzi, S.M., Stubbs, M., and Like, A.A. 1993. The BBZ/Wor rat: clinical characteristics of the diabetic syndrome. Diabetologia 36: 912-919.

5. Ikeda, H., Shino, A., Matsuo, T., Iwatsuka, H., and Suzuoki, Z. 1981. A new genetically obese-hyperglycemic rat (Wistar Fatty). Diabetes 30: 1045-1050.

6. Ishizuka, T., Ernsberger, P., Liu, S., Bedol, D., Lehman, T.M., Koletsky, R.J., and Friedman, J.E. 1998. Phenotypic consequences of a nonsense mutation in the leptin receptor gene $\left(f a^{k}\right)$ in obese spontaneously hypertensive Koletsky rats (SHROB). J. Nutr. 128: 2299-2306.

7. Kava, R.A., West, D.D., Lukasik, V.A., and Greenwood, M.R.C. 1989. Sexual dimorphism of hyperglycemia and glucose tolerance in Wistar fatty rats. Diabetes 38: 159163.

8. Kitada, K., Voigt, B., Kondo, Y., and Serikawa, T. 2000. An integrated rat genome map based on genetic and cytogenetic data. Exp. Anim. 49: 119-126.

9. Markel, P., Shu, P., Ebeling, C., Carlson, G.A., Nagle, D.L., Smutko, J.S., and Moore K.J. 1997. Theoretical and empirical issues for marker-assisted breeding of congenic mouse strains. Nat. Genet. 17: 280-284.

10. Masuyama, T., Ishibiki, J., Awata, T., Noda, M., Kanazawa, Y., Sugawara, M., and Komeda, K. 2000. An improved genetic linkage map of the rat chromosome 20. Comp. Med. 50: 369-373.

11. Masuyama, T., Fuse, M., Yokoi, N., Shinohara, M., Tsujii, H., Kanazawa, M., Kanazawa, Y., Komeda, K., and Taniguchi, K. 2003. Genetic analysis for diabetes in a new rat model of nonobese type 2 diabetes, Spontaneously Diabetic Torii rat. Biochem. Biophys. Res. Commun. 304: 196-206.

12. Masuyama, T., Komeda, K., Hara, A., Noda, M., Shinohara, M., Oikawa, T., Kanazawa, Y., and Taniguchi, K. 2004. Chronological characterization of diabetes development in male Spontaneously Diabetic Torii rats. Biochem. Biophys. Res. Commun. 314: 870-877.

13. Mu, J.L., Naggert, J.K., Svenson, K.L., Collin, G.B., Kim, J.H., McFarland, C., Nishina, P.M., Levine, D.M., Williams, K.J., and Paigen, B. 1999. Quantitative trait loci analysis for the differences in susceptibility to atherosclerosis and diabetes between inbred mouse strains C57BL/6J and C57BLKS/J. J. Lipid Res. 40: 1328-1335.

14. Nathan, D.M. and Cagliero, E. 2001. Diabetes mellitus. pp. 827-926. In: Endocrinology and Metabolism. Felig, P. and Frohman, L.A. (eds), 4th edn, McGraw-Hill, USA.

15. Peterson, R.G., Shaw, M.W., Neel, M-A., and Eichberg, J. 1990. Zucker diabetic fatty rat as model for non-insulindependent diabetes mellitus. ILAR News 32: 16-19.

16. Pralong, F.P. and Gaillard, R.C. 2001. Neuroendocrine effects of leptin. Pituitary 4: 25-32.

17. Shinohara, M., Masuyama, T., Shoda, T., Takahashi, T., 
Katsuda, Y., Komeda, K., Kuroki, M., Kakehashi, A., and Kanazawa, Y. 2000. A new spontaneously diabetic nonobese Torii rat strain with severe ocular complications. Int. J. Exp. Diabetes Res. 1: 89-100.

18. Takaya, K., Ogawa, Y., Isse, N., Okazaki, T., Satoh, N., Masuzaki, H., Mori, K., Tamura, N., Hosoda, K., and Nakao, K. 1996. Molecular cloning of rat leptin receptor isoform complementary DNAs-identification of a missense mutation in Zucker fatty ( $f a / f a)$ rats. Biochem. Biophys. Res. Commun. 225: 75-83.

19. Terauchi, Y., Matsui, J., Suzuki, R., Kubota, N., Komeda, K., Aizawa, S., Eto, K., Kimura, S., Nagai, R., Tobe, K., Lienhard, G.E., and Kadowaki, T. 2003. Impact of genetic background and ablation of insulin receptor substrate (IRS)3 on IRS-2 knock-out mice. J. Biol. Chem. 278:
14284-14290.

20. The Expert Committee on the Diagnosis and Classification of Diabetes Mellitus. 1997. Report of the expert committee on the diagnosis and classification of diabetes mellitus. Diabetes Care 20: 1183-1197.

21. Weir, G.C., Laybutt, D.R., Kaneto, H., Bonner-Weir, S., and Sharma, A. 2001. $\beta$-cell adaptation and decompensation during the progression of diabetes. Diabetes 50: S154S159.

22. Zabeau, L., Lavens, D., Peelman, F., Eyckerman, S., Vandekerckhove, J., and Tavernier, J. 2003. The ins and outs of leptin receptor activation. FEBS Lett. 546: 45-50.

23. Zucker, L.M. and Antoniades, H.N. 1972. Insulin and obesity in the Zucker genetically obese rat "fatty". Endocrinology 90: 1320-1330. 